\title{
Accuracy of Dose Delivery in Multiple Breath-Hold Segmented Volumetric Modulated Arc Therapy: A Static Phantom Study
}

\author{
Kimiya Noto, ${ }^{1}$ Shinichi Ueda, ${ }^{1}$ Hironori Kojima, ${ }^{1}$ Naoki Isomura, ${ }^{1}$ Akihiro Takemura, \\ Shigeyuki Takamatsu, ${ }^{3}$ Tomoyasu Kumano, ${ }^{3}$ and Tsuyoshi Takanaka ${ }^{4}$ \\ ${ }^{1}$ Department of Radiology, Kanazawa University Hospital, 13-1 Takara-machi, Kanazawa, Ishikawa 920-8641, Japan \\ ${ }^{2}$ Faculty of Health Sciences, Institute of Medical Pharmaceutical and Health Sciences, Kanazawa University, 5-11-80 Kodatsuno, \\ Kanazawa, Ishikawa 920-0942, Japan \\ ${ }^{3}$ Department of Radiotherapy, Graduate School of Medical Science, Kanazawa University, 13-1 Takara-machi, Kanazawa, \\ Ishikawa 920-8641, Japan \\ ${ }^{4}$ Department of Radiation Oncology, Kouseiren Takaoka Hospital, 5-10 Eirakucho, Takaoka, Toyama 933-8555, Japan
}

Correspondence should be addressed to Kimiya Noto; kimiyanoto@hotmail.com

Received 6 June 2014; Accepted 9 September 2014; Published 16 September 2014

Academic Editor: Oliver Micke

Copyright (C) 2014 Kimiya Noto et al. This is an open access article distributed under the Creative Commons Attribution License, which permits unrestricted use, distribution, and reproduction in any medium, provided the original work is properly cited.

Purpose. Accuracy of dose delivery in multiple breath-hold segmented volumetric modulated arc therapy (VMAT) was evaluated in comparison to noninterrupted VMAT using a static phantom. Material and Methods. Five VMAT plans were evaluated. A Synergy linear accelerator (Elekta AB, Stockholm, Sweden) was employed. A VMAT delivery sequence was divided into multiple segments according to each of the predefined breath-hold periods (10, 15, 20, 30, and 40 seconds). The segmented VMAT delivery was compared to noninterrupted VMAT delivery in terms of the isocenter dose and pass rates of a dose difference of $1 \%$ with a dose threshold of $10 \%$ of the maximum dose on a central coronal plane using a two-dimensional dosimeter, MatriXX Evolution (IBA Dosimetry, Schwarzenbruck, Germany). Results. Means of the isocenter dose differences were $0.5 \%, 0.2 \%, 0.2 \%, 0.0 \%$, and $0.0 \%$ for the beam-on-times between interrupts of 10, 15, 20,30, and 40 seconds, respectively. Means of the pass rates were $85 \%, 99.9 \%$, $100 \%, 100 \%$, and $100 \%$ in the same order as the above. Conclusion. Our static phantom study indicated that the multiple breath-hold segmented VMAT maintains stable and accurate dose delivery when the beam-on-time between interrupts is 15 seconds or greater.

\section{Introduction}

Although radiotherapy has been successfully applied to lung, liver, and pancreas tumors [1-3], breathing motion needs to be considered for the tumors located in proximity to the diaphragm [4-9]. A traditional approach is an enlarged internal margin that was added to a gross tumor volume (GTV) or a clinical target volume (CTV), resulting in possible higher complication to neighboring organs at risk (OARs) [10].

To minimize the internal margin, breath-hold with active breathing control $(\mathrm{ABC})$ or patient voluntary breath-hold was used for intensity modulated radiation therapy (IMRT) $[4,10-12]$ among other techniques such as gating. IMRT provides more conformal dose for the target and more reduced dose for OARs compared to 3D conformal radiotherapy (3D-CRT). A disadvantage of IMRT is increased monitor units and thus beam-on-time, thereby possibly causing larger intrafractional tumor localization error [13, 14].

Volumetric modulated arc therapy (VMAT) allows a faster dose delivery while gantry and multileaf collimator (MLC) are dynamically controlled [15-19]. A combination of breath-hold and VMAT may lead to a quick and accurate treatment option for a moving tumor close to diaphragm. Nevertheless, the beam-on-time for a VMAT delivery is typically two to four minutes, thereby preventing a single breath-hold treatment.

A natural solution of this problem may be a segmented breath-hold VMAT delivery having a number of breath-hold segments. However, accuracy of delivered dose in segmented breath-hold VMAT has not been reported. The purpose of this study was evaluation of delivered dose accuracy for segmented breath-hold VMAT. 
TABLE 1: The monitor units per fraction, the gantry arc angles, and the beam-on-times required for noninterrupted delivery are shown for five VMAT clinical cases. When the beams were interrupted for five seconds with varied beam-on-times between interrupts, the number of VMAT segments was counted and recorded.

\begin{tabular}{|c|c|c|c|c|c|c|c|c|}
\hline \multirow[t]{2}{*}{ Case } & \multirow[t]{2}{*}{$\mathrm{MU} / \mathrm{fx}$} & \multirow[t]{2}{*}{$\begin{array}{l}\text { Gantry arc angle } \\
\text { (degree) }\end{array}$} & \multirow[t]{2}{*}{ Beam-on-time (s) } & \multicolumn{5}{|c|}{$\begin{array}{l}\text { Measured number of VMAT segments } \\
\text { Beam-on-time between interrupts (s) }\end{array}$} \\
\hline & & & & 10 & 15 & 20 & 30 & 40 \\
\hline 1 & 514.4 & 195 & 210 & 29 & 17 & 12 & 8 & 6 \\
\hline 2 & 390.7 & 160 & 97 & 14 & 8 & 6 & 4 & 3 \\
\hline 3 & 588.5 & 230 & 137 & 21 & 12 & 9 & 6 & 4 \\
\hline 4 & 786.3 & 360 & 214 & 32 & 18 & 13 & 8 & 6 \\
\hline 5 & 544.2 & 360 & 198 & 30 & 17 & 12 & 8 & 6 \\
\hline
\end{tabular}

\section{Material and Methods}

2.1. Linear Accelerator and Treatment Planning System. A Synergy linear accelerator (Elekta AB, Stockholm, Sweden) with a multileaf collimator of $4 \mathrm{~mm}$ leaf width was used. A photon energy of $6 \mathrm{MV}$ was selected. Monaco treatment planning system (Elekta AB, Stockholm, Sweden) was employed and dose calculation was performed by a built-in Monte Carlo algorithm. As a convergence criterion, a standard deviation of $3 \%$ was adopted with a dose grid size of $2 \mathrm{~mm}$ for this phantom study.

Five VMAT plans (three for liver and two for lung) were randomly chosen from previously delivered clinical plans, and accuracy of dose delivery in multiple breath-hold segmented VMAT was evaluated in comparison to noninterrupted VMAT. Prior to conducting the present study, dose verification for the original five VMAT plans had been performed using EDR2 films (Kodak, Rochester, USA) and a film scanner, DD system (R-Tech, Tokyo, Japan), leading to mean gamma index pass rate of $98.9 \%$ (range: 97.8 to $99.7 \%$ ) under a criterion of $3 \mathrm{~mm} / 3 \%$ (normalized to locally measured dose) on a central coronal plane with a dose threshold of $50 \%$ of the maximum planer dose, whereas mean isocenter dose discrepancy between the plans and the measurements was $0.5 \%$ with a range of -0.6 to $2.3 \%$.

2.2. 2D Ionization Chamber Array System. A two-dimensional dose detector, MatriXX Evolution (IBA Dosimetry, Schwarzenbruck, Germany), was used, which had 1020 ionization microchambers distributed in an active area of $24 \times$ $24 \mathrm{~cm}^{2}$. Each chamber had a diameter of $4.5 \mathrm{~mm}$, a height of $5 \mathrm{~mm}$, and a volume of $0.08 \mathrm{~cm}^{3}$, with chamber spacing of $7.62 \mathrm{~mm}$. MatriXX was widely used for dose verification studies [20-24]. In this experiment, MatriXX was positioned at the center of a plastic water phantom, MULTICube (IBA Dosimetry, Schwarzenbruck, Germany) having a length of $31.4 \mathrm{~cm}$, a width of $34 \mathrm{~cm}$, and a height of $22 \mathrm{~cm}$. Measurement and subsequent data analysis were performed by OmniPro-I'mRT 1.5a (IBA Dosimetry, Schwarzenbruck, Germany) with a data sampling interval of $50 \mathrm{~ms}$. The MatriXX needs to be calibrated for absolute dose measurement as follows. After placing the MatriXX in the MULTICube phantom, photons with energy of $6 \mathrm{MV}$ and a field size of $10 \times$ $10 \mathrm{~cm}^{2}$ were employed. Cross-calibration was performed between each of the central four detectors of the MatriXX and an ionization chamber placed at each of the four detector positions, where a Farmer chamber (30013, PTW, Freiburg, Germany) traceable to national standards was inserted into another water equivalent phantom with a size identical to the MatriXX.

\subsection{Dose Comparison of Multiple Breath-Hold VMAT Plans} and Noninterrupted Plans. Five different beam-on-times between interrupts of 10,15, 20, 30, and 40 seconds were predefined as the elapsed time from pushing the MV beam start button to pushing the MV interrpt button, which may represent typical breath-hold periods. Manually interrupted beam-off period was maintained for five seconds for all the measurements. A VMAT delivery sequence was divided into multiple segments according to each breath-hold period. The segmented VMAT delivery was compared to noninterrupted VMAT delivery in terms of the isocenter dose and the pass rates of a dose difference of $1 \%$ with a dose threshold of $10 \%$ of the maximum dose on a central coronal plane. The position of the MatriXX kept unchanged throughout this measurement. The isocenter dose was calculated by averaging doses measured by the central four chambers. Percentage difference (PD) in the isocenter dose was calculated by (1), where Dose $_{\text {segmented }}$ is an isocenter dose resulting from the seg-

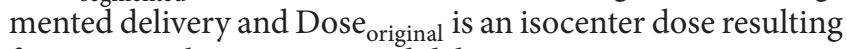
from original noninterrupted delivery:

$$
\mathrm{PD}=\frac{\text { Dose }_{\text {segmented }}-\text { Dose }_{\text {original }}}{\text { Dose }_{\text {original }}} \times 100 .
$$

\section{Results}

Table 1 shows the monitor units per fraction, the gantry arc angles, and the beam-on-times required for noninterrupted delivery for five VMAT clinical cases. When the beams were interrupted for five seconds with varied beam-on-times between interrupts, the number of VMAT segments was counted and recorded. When the beam-on-time between interrupts was reduced to ten seconds, the number of VMAT segments was highly increased because beams were always delivered after a few seconds of preparation. Table 2 shows the mean, the SD, the minimum, and the maximum of the 
TABLE 2: The mean, the SD, the minimum, and the maximum of the segmental MUs for the five cases with the five different beam-ontimes between interrupts.

\begin{tabular}{|c|c|c|c|c|c|}
\hline $\begin{array}{l}\text { Beam-on-time } \\
\text { between interrupts (s) }\end{array}$ & Case & Mean & $\mathrm{SD}$ & Min. & Max. \\
\hline \multirow{5}{*}{10} & 1 & 17.5 & 6.6 & 8.9 & 36 \\
\hline & 2 & 27.9 & 4.6 & 15.7 & 31.1 \\
\hline & 3 & 28.0 & 7.0 & 10.2 & 38.6 \\
\hline & 4 & 24.6 & 10.9 & 12.6 & 55.8 \\
\hline & 5 & 19.1 & 5.9 & 11.4 & 40.3 \\
\hline \multirow{5}{*}{15} & 1 & 30.3 & 11.0 & 14.5 & 54.0 \\
\hline & 2 & 48.8 & 9.3 & 26.9 & 57.6 \\
\hline & 3 & 49.0 & 10.7 & 34.0 & 65.3 \\
\hline & 4 & 43.7 & 16.9 & 25.1 & 83.6 \\
\hline & 5 & 32.6 & 8.7 & 20.0 & 51.2 \\
\hline \multirow{5}{*}{20} & 1 & 42.9 & 18.8 & 26.5 & 91.4 \\
\hline & 2 & 65.1 & 20.5 & 23.9 & 79.0 \\
\hline & 3 & 65.3 & 21.9 & 12.8 & 80.7 \\
\hline & 4 & 60.5 & 25.3 & 25.8 & 112.1 \\
\hline & 5 & 46.2 & 13.1 & 29.4 & 75.0 \\
\hline \multirow{5}{*}{30} & 1 & 64.3 & 19.8 & 39.8 & 102.1 \\
\hline & 2 & 97.7 & 40.5 & 37.4 & 124.3 \\
\hline & 3 & 117.7 & 21.8 & 94.2 & 149.2 \\
\hline & 4 & 98.3 & 38.0 & 63.9 & 171.2 \\
\hline & 5 & 69.3 & 22.8 & 25.4 & 97.7 \\
\hline \multirow{5}{*}{40} & 1 & 85.7 & 22.0 & 63.7 & 119.7 \\
\hline & 2 & 130.2 & 56.3 & 65.4 & 166.3 \\
\hline & 3 & 147.1 & 25.8 & 115.5 & 178.6 \\
\hline & 4 & 131.1 & 53.5 & 75.0 & 220.7 \\
\hline & 5 & 110.8 & 17.3 & 92.2 & 138.0 \\
\hline
\end{tabular}

segmental MUs for the five different beam-on-times between interrupts.

Figure 1 depicts plots of isocenter dose differences from noninterrupted delivery for the five cases as a function of the beam-on-time between interrupts. Each circle shows each mean of the five cases, and a range is also shown as a vertical line. Means and ranges (in parentheses) among the five plans were $0.5 \%$ ( 0 to $1.3 \%$ ), $0.2 \%$ ( -0.1 to $0.6 \%$ ), $0.2 \%$ $(-0.2$ to $0.4 \%), 0.0 \%(-0.1$ to $0.2 \%)$, and $0.0 \%(-0.1$ to $0.1 \%$ ) for beam-on-times between interrupts of $10,15,20,30$, and 40 seconds, respectively. The average of isocenter dose differences was gradually reduced when the beam-on-time between interrupts was increased; however, the differences were not significant due to relatively large ranges in each plot.

Figure 2 shows plots of pass rates of $1 \%$ dose difference from noninterrupted delivery on a central coronal plane for the five cases as a function of the beam-on-time between interrupts. The dose difference was normalized to each local dose of noninterrupted delivery (as shown in (1)), and a dose threshold of $10 \%$ of the maximum dose on the coronal plane was employed for the pass rate calculation. Each circle shows each mean of the five cases, and a vertical line indicates

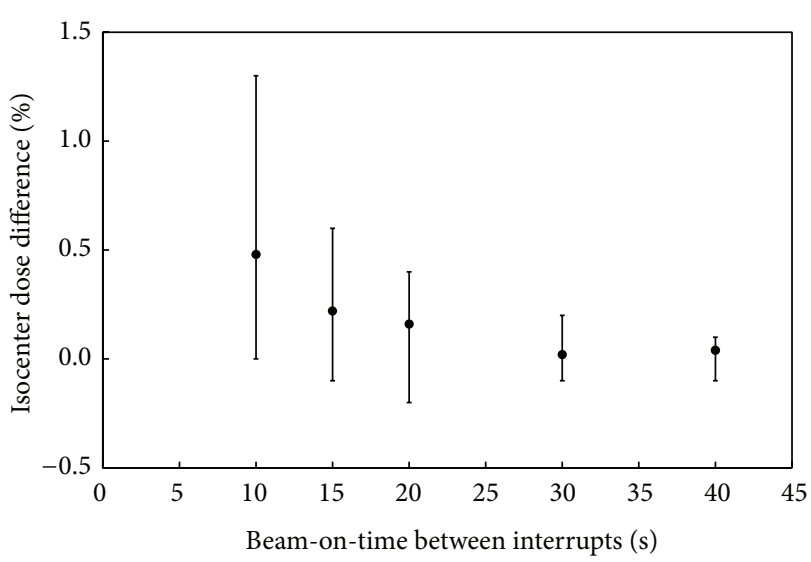

FIgURE 1: Plots of isocenter dose differences from noninterrupted delivery for the five cases as a function of beam-on-time between interrupts. Each circle shows each mean of the five cases, and a range is also shown as a vertical line.



FIgURE 2: Plots of pass rates of $1 \%$ dose difference from noninterrupted delivery on a central coronal plane for the five cases as a function of beam-on-time between interrupts. The dose difference was normalized to each local dose with no interrupt, and a dose threshold of $10 \%$ of the maximum dose on the coronal plane was employed for the pass rate calculation. Each circle shows each mean of the five cases, and a vertical line indicates a range, where most of the ranges were too small to be visualized.

a range, where most of the ranges were too small to be visualized. In detail, means and ranges (in parentheses) of the pass rates of the dose difference of $1 \%$ were $85 \%$ (61 to $100 \%$ ), $99.9 \%$ (99.5 to $100 \%$ ), $100 \%$ (100 to $100 \%$ ), 100\% (100 to $100 \%$ ), and $100 \%$ (100 to $100 \%$ ) in the same order shown in Figure 1, indicating that segmented VMAT maintains stable and accurate dose delivery when the beam-on-time between interrupts is 15 seconds or greater.

It was also confirmed that, when the beam-on-time between interrupts was 10 seconds, the worse pass rates were observed in cases 1 and 5, where the mean MUs were lower than others as shown in Table 2. 


\section{Discussion}

Compared to step and shoot and dynamic IMRT deliveries, VMAT delivery is much quicker with decreased monitor units. However, breathing motion needs to be considered for lower-lobe lung, liver, and pancreas tumors located close to the diaphragm. Mori et al. [7] reported that the breathing amplitude for a pancreas cancer in the craniocaudal direction was approximately $10 \mathrm{~mm}$. Besides, it is well known that the internal margin can be reduced under constrained breathing conditions [25-29].

The segmented breath-hold VMAT technique is regarded as a novel treatment option. The number of breath-hold segments depends on gantry speeds during delivery, total monitor units, and tolerable breath-hold periods for each patient. Dose delivery should not be less accurate when segmented VMAT is employed. This was our motivation of this study, and accuracy of the segmented breath-hold VMAT delivery was evaluated with varied beam-on-times between interrupts. The two-dimensional ion chamber array, MatriXX, employed for this study allowed measurements for absolute dose and dose distributions at the same time, facilitating an efficient quality assurance procedure even under a limited spatial resolution [21-24]. Amerio et al. [20] reported that the MatriXX resulted in good dose linearity with no dose rate dependence, and the dose discrepancy against Farmer chamber reading was less than $0.5 \%$. In our study, the maximum isocenter dose discrepancies against original noninterrupted VMAT plans were $1.3 \%$ for a beamon-time between interrupts of 10 seconds. The discrepancy was decreased down to $0.6 \%$ or less for the segmented beamon-time of 15 seconds or larger. According to American Association of Physicists in Medicine (AAPM) Task Group report 53 [30], tolerance of absolute dosimetry using an ionization chamber was less than $5 \%$. The discrepancy we obtained was much less than the AAPM recommendation, and, therefore, the segmented breath-hold VMAT delivery is regarded as sufficiently accurate.

Meanwhile, the pass rates of the dose difference on the central coronal plane were nearly $100 \%$ when the segmented beam-on-time was 15 seconds or greater. It is anticipated that the gantry inertia may require a significant transient period for gantry speed rise when the gantry restarts rotating after each beam interrupt. The dose rate may need to be adjusted during this period in order to provide accurate MU/degree specified by the treatment planning system. In our current linac controller, the dose rate changes stepwise by a factor of two, possibly leading to limited performance for the MU/ degree adjustment during this transient period. When the beam-on-time between interrupts is reduced, the impact of the dose contribution during this transient period on the total dose may be increased, thereby decreasing the total dose accuracy. Based on the results obtained, it is suggested that the breath-hold time of 15 seconds or longer may be appropriate to maintain stable delivery.

The limitation of this study is that a static phantom was used for evaluating the delivery accuracy; in other words, the impact of the reproducibility of the target location on dose distributions for multiple breath-hold VMAT was not evaluated, which requires further investigation.

\section{Conclusion}

We have evaluated accuracy of dose delivery in multiple breath-hold segmented VMAT using a static phantom, showing that the segmented VMAT maintains stable and accurate dose delivery when the beam-on-time between interrupts is 15 seconds or greater. It is expected that the segmented breath-hold VMAT delivery technique may provide an accurate and customizable treatment option based on breathing conditions for each patient.

\section{Conflict of Interests}

The authors declare that there is no conflict of interests regarding the publication of this paper.

\section{References}

[1] E. Ben-Josef, A. F. Shields, U. Vaishampayan et al., "Intensitymodulated radiotherapy (IMRT) and concurrent capecitabine for pancreatic cancer," International Journal of Radiation Oncology Biology Physics, vol. 59, no. 2, pp. 454-459, 2004.

[2] P. Merle, F. Mornex, and C. Trepo, "Innovative therapy for hepatocellular carcinoma: three-dimensional high-dose photon radiotherapy," Cancer Letters, vol. 286, no. 1, pp. 129-133, 2009.

[3] A. Bezjak, R. B. Rumble, G. Rodrigues, A. Hope, and P. Warde, "Intensity-modulated radiotherapy in the treatment of lung cancer," Clinical Oncology, vol. 24, no. 7, pp. 508-520, 2012.

[4] L. A. Dawson, K. K. Brock, S. Kazanjian et al., "The reproducibility of organ position using active breathing control (ABC) during liver radiotherapy," International Journal of Radiation Oncology Biology Physics, vol. 51, no. 5, pp. 1410-1421, 2001.

[5] B. Bussels, L. Goethals, M. Feron et al., "Respiration-induced movement of the upper abdominal organs: a pitfall for the threedimensional conformal radiation treatment of pancreatic cancer," Radiotherapy and Oncology, vol. 68, no. 1, pp. 69-74, 2003.

[6] E. D. Brandner, A. Wu, H. Chen et al., "Abdominal organ motion measured using 4D CT," International Journal of Radiation Oncology, Biology, Physics, vol. 65, no. 2, pp. 554-560, 2006.

[7] S. Mori, R. Hara, T. Yanagi et al., "Four-dimensional measurement of intrafractional respiratory motion of pancreatic tumors using a 256 multi-slice CT scanner," Radiotherapy and Oncology, vol. 92, no. 2, pp. 231-237, 2009.

[8] N. Katoh, R. Onimaru, Y. Sakuhara et al., "Real-time tumortracking radiotherapy for adrenal tumors," Radiotherapy and Oncology, vol. 87, no. 3, pp. 418-424, 2008.

[9] A. S. Pai Panandiker, S. Sharma, M. H. Naik et al., "Novel assessment of renal motion in children as measured via fourdimensional computed tomography," International Journal of Radiation Oncology Biology Physics, vol. 82, no. 5, pp. 1771-1776, 2012.

[10] G. Z. Gong, Y. Yin, L. G. Xing et al., "RapidArc combined with the active breathing coordinator provides an effective and accurate approach for the radiotherapy of hepatocellular carcinoma," Strahlentherapie und Onkologie, vol. 188, no. 3, pp. 262-268, 2012.

[11] W. $\mathrm{Hu}, \mathrm{J}$. Ye, J. Wang, Q. Xu, and Z. Zhang, "Incorporating breath holding and image guidance in the adjuvant gastric cancer radiotherapy: a dosimetric study," Radiation Oncology, vol. 7, no. 1, article 98, 2012. 
[12] M. Nakamura, S. Kishimoto, K. Iwamura et al., "Dosimetric investigation of breath-hold intensity-modulated radiotherapy for pancreatic cancer," Medical Physics, vol. 39, no. 1, pp. 48-54, 2012.

[13] J. Z. Wang, X. A. Li, W. D. D’Souza, and R. D. Stewart, "Impact of prolonged fraction delivery times on tumor control: a note of caution for intensity-modulated radiation therapy (IMRT)," International Journal of Radiation Oncology Biology Physics, vol. 57, no. 2, pp. 543-552, 2003.

[14] D. P. Gierga, G. T. Y. Chen, J. H. Kung, M. Betke, J. Lombardi, and C. G. Willett, "Quantification of respiration-induced abdominal tumor motion and its impact on IMRT dose distributions," International Journal of Radiation Oncology Biology Physics, vol. 58, no. 5, pp. 1584-1595, 2004.

[15] C. C. Popescu, I. A. Olivotto, W. A. Beckham et al., "Volumetric modulated arc therapy improves dosimetry and reduces treatment time compared to conventional Intensity-Modulated radiotherapy for locoregional radiotherapy of left-sided breast cancer and internal mammary nodes," International Journal of Radiation Oncology Biology Physics, vol. 76, no. 1, pp. 287-295, 2010.

[16] D. Wolff, F. Stieler, B. Hermann et al., "Clinical implementation of volumetric intensity-modulated arc therapy (VMAT) with ERGO++," Strahlentherapie und Onkologie, vol. 186, no. 5, pp. 280-288, 2010.

[17] W. Eppinga, F. Lagerwaard, W. Verbakel, B. Slotman, and S. Senan, "Volumetric modulated arc therapy for advanced pancreatic cancer," Strahlentherapie und Onkologie, vol. 186, no. 7, pp. 382-387, 2010.

[18] F. Stieler, D. Wolff, L. Bauer, H.-J. Wertz, F. Wenz, and F. Lohr, "Reirradiation of spinal column metastases: comparison of several treatment techniques and dosimetric validation for the use of VMAT,' Strahlentherapie und Onkologie, vol. 187, no. 7, pp. 406-415, 2011.

[19] Y. Yin, C. Ma, M. Gao et al., "Dosimetric comparison of rapidarc with fixed gantry intensity-modulated radiotherapy treatment for multiple liver metastases radiotherapy," Medical Dosimetry, vol. 36 , no. 4 , pp. 448-454, 2011.

[20] S. Amerio, A. Boriano, F. Bourhaleb et al., "Dosimetric characterization of a large area pixel-segmented ionization chamber," Medical Physics, vol. 31, no. 2, pp. 414-420, 2004.

[21] T. Wiezorek, N. Banz, M. Schwedas et al., "Dosimetric quality assurance for intensity-modulated radiotherapy: feasibility study for a filmless approach," Strahlentherapie und Onkologie, vol. 181, no. 7, pp. 468-474, 2005.

[22] E. Spezi, A. L. Angelini, F. Romani, and A. Ferri, "Characterization of a 2D ion chamber array for the verification of radiotherapy treatments," Physics in Medicine and Biology, vol. 50, no. 14, pp. 3361-3373, 2005.

[23] B. Poppe, A. Blechschmidt, A. Djouguela et al., "Two-dimensional ionization chamber arrays for IMRT plan verification," Medical Physics, vol. 33, no. 4, pp. 1005-1015, 2006.

[24] B. Poppe, A. Djouguela, A. Blechschmidt, K. Willborn, A. Rühmann, and D. Harder, "Spatial resolution of 2D ionization chamber arrays for IMRT dose verification: Single-detector size and sampling step width," Physics in Medicine and Biology, vol. 52, pp. 2921-2935, 2007.

[25] H. Onishi, K. Kuriyama, T. Komiyama et al., "CT evaluation of patient deep inspiration self-breath-holding: how precisely can patients reproduce the tumor position in the absence of respiratory monitoring devices?" Medical Physics, vol. 30, no. 6, pp. 1183-1187, 2003.
[26] H. Onishi, H. Kawakami, K. Marino et al., "A simple respiratory indicator for irradiation during voluntary breath holding: a onetouch device without electronic materials," Radiology, vol. 255, no. 3, pp. 917-923, 2010.

[27] J. W. Wong, M. B. Sharpe, D. A. Jaffray et al., "The use of active breathing control (ABC) to reduce margin for breathing motion," International Journal of Radiation Oncology Biology Physics, vol. 44, no. 4, pp. 911-919, 1999.

[28] T. I. Tarohda, M. Ishiguro, K. Hasegawa et al., "The management of tumor motions in the stereotactic irradiation to lung cancer under the use of Abches to control active breathing," Medical Physics, vol. 38, no. 7, pp. 4141-4146, 2011.

[29] M. Nakamura, K. Shibuya, T. Shiinoki et al., "Positional reproducibility of pancreatic tumors under end-exhalation breathhold conditions using a visual feedback technique," International Journal of Radiation Oncology Biology Physics, vol. 79, no. 5, pp. 1565-1571, 2011.

[30] B. Fraass, K. Doppke, M. Hunt et al., "American association of physicists in medicine radiation therapy committee task group 53: quality assurance for clinical radiotherapy treatment planning," Medical Physics, vol. 25, no. 10, pp. 1773-1829, 1998. 


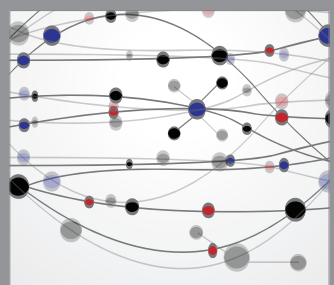

The Scientific World Journal
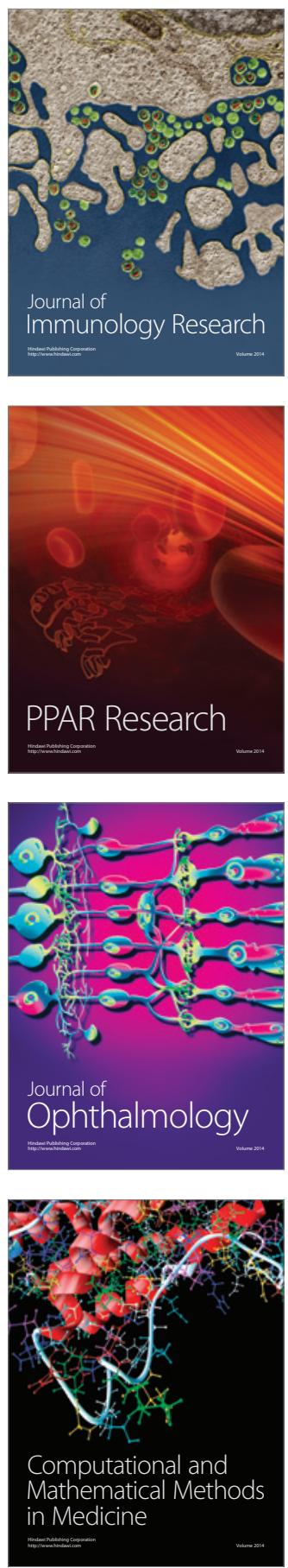

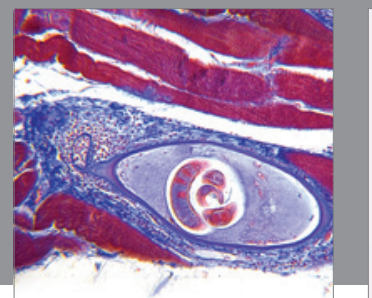

Gastroenterology

Research and Practice
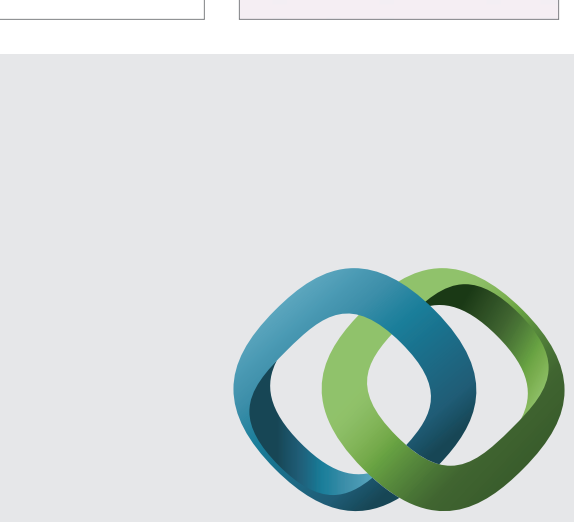

\section{Hindawi}

Submit your manuscripts at

http://www.hindawi.com
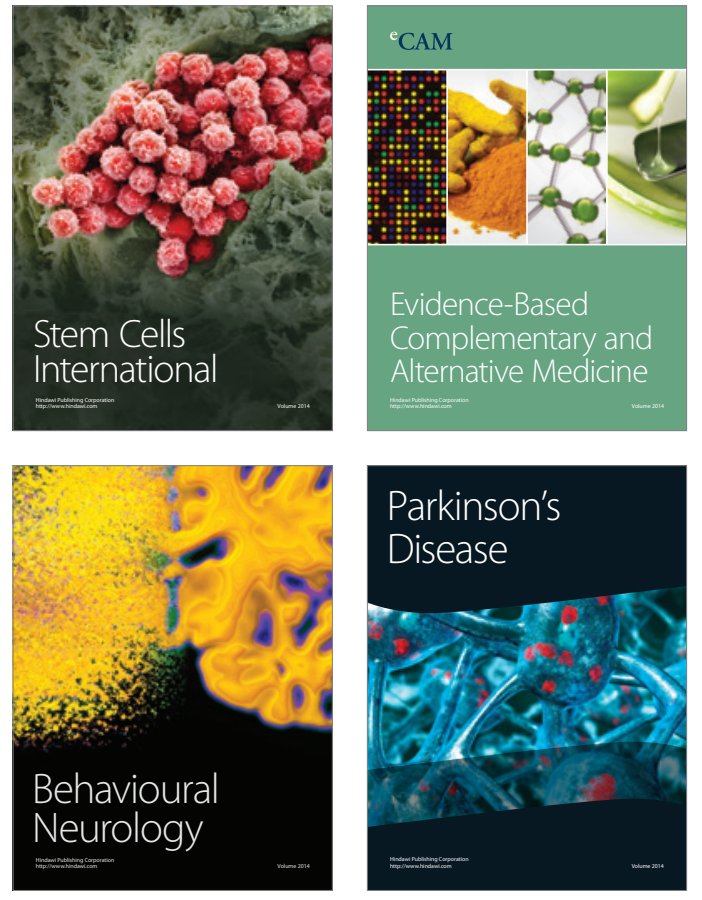
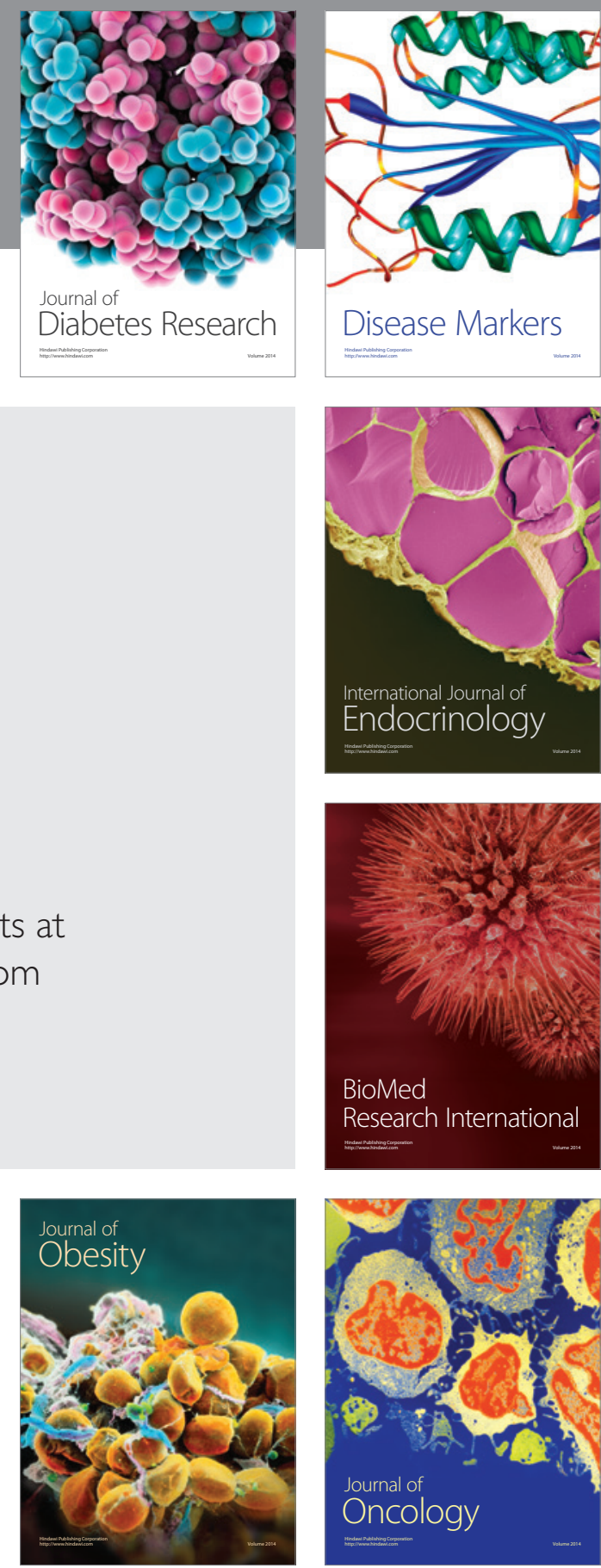

Disease Markers
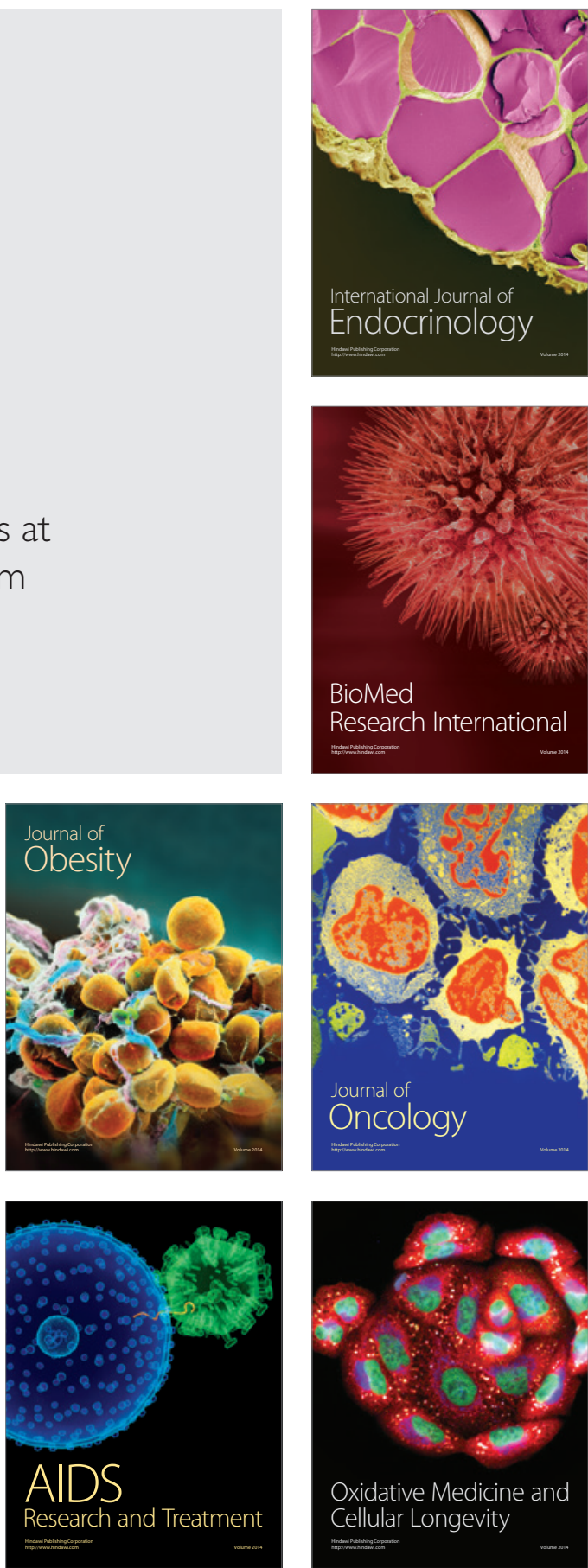